The Astrophysical Journal Supplement Series, 90:905-907, 1994 February

O 1994. The American Astronomical Society. All rights reserved. Printed in U.S.A.

\title{
POLARIZED ONE-QUANTUM ANNIHILATION IN STRONG MAGNETIC FIELDS AS A PROCESS FOR PARTICLE DECELERATION IN NEUTRON STAR ATMOSPHERES
}

\author{
D. BERG, M. MENTZEL, AND G. WUNNER \\ Theoretische Physik I, Ruhr-Universität Bochum, 44780 Bochum, Universitätsstrasse 150, Germany \\ Received 1993 February 28; accepted 1993 August 12
}

\begin{abstract}
Since several gamma-ray bursts seem to have their origin in the vicinity of neutron stars, where strong magnetic fields are present $\left(B \simeq 10^{8} T\right.$ ), electrons and positrons have to be described, in this instance, by relativistic Landau states. As is well known, strong magnetic fields produce important effects: motion perpendicular to the field is quantized, transverse momentum is not conserved, and polarization effects become important. Moreover, in contrast to the field-free case, exotic processes such as $1 \gamma$ pair-annihilation are not forbidden. With growing magnetic field, the cross section of this process becomes more and more nonnegligible. This cross section strongly depends on the polarization of the electron-positron pairs. Thus the deceleration via annihilation turns out to be a strongly polarization-dependent phenomenon in magnetized electron-positron plasmas.
\end{abstract}

Subject headings: acceleration of particles — gamma rays: theory — polarization radiation mechanisms: nonthermal — stars: neutron

In high-energy astrophysics, recent interest is concentrated on objects with superstrong magnetic fields, such as a broad class of neutron stars with $B \simeq 10^{8} T$. Gamma-ray bursts (GRBs), very short intense and unpredictable phenomena which produce mainly radiation of more than $1 \mathrm{MeV}$, are believed to originate at least partially in the vicinity of these highly magnetized compact objects (Harding 1991). The radiation is produced in this high-field environment by a number of elementary processes in the neutron star magnetosphere (Herold, Ruder, \& Wunner 1981). Particles are accelerated by the absorption of $\gamma$-quanta, and the particle plasma is again cooled due to the emission of photons. Processes involving electrons, positrons, and photons such as scattering, electron-positron annihilation, and cyclotron absorption should contribute dominantly to the GRB-spectra (Mazets et al. 1981). In order to describe the high-energy spectra, one has to perform the fully relativistic analysis of the quantum-electrodynamical processes in these high magnetic fields (Melrose \& Parle 1983).

To obtain the electron and positron states for calculating the relevant cross sections, we solve Dirac's equation in a uniform time-independent magnetic field:

$$
(\text { ( }-m) \Psi=0,
$$

where the momentum operator in the minimal substitution is given by

$$
\Pi^{\mu}=i \partial^{\mu}+e A^{\mu} .
$$

To have the magnetic field orientation in the $z$-direction, we take the external field in the Landau gauge

$$
A^{\mu}=(0,0, B x, 0) \text {. }
$$

The Dirac equation can be solved analytically and yields the well-known eigenvalues

$$
E_{N, p_{z}}= \pm m \sqrt{1+2 N B / B_{\mathrm{cr}}+\left(p_{z} / m\right)^{2}} .
$$

Here, $B$ is written in units of the critical field where the Larmor length equals the electron Compton length

$$
B_{\mathrm{cr}}=\frac{m^{2}}{e}=4.41 * 10^{9} \mathrm{~T},
$$

$N$ denotes the Landau quantum number of the state, and $p$, is the projection of momentum into the $z$-direction. It is clear that the energy spectrum is continuous in $p_{z}$, and discrete in Landau levels $(N=0,1, \cdots)$ like in atomic spectra.

The wavefunctions for electron and positron are obtained with the following Ansatz

$$
\Psi\left(x^{\mu}\right)=e^{i\left(p_{y} y+p_{z} z-E t\right)} f(x) .
$$

In our calculation, we use the wavefunctions of Sokolov \& Ternov (1968), which are simultaneously eigenstates of the magnetic moment operator and are relativistically covariant. The photon states are the usual field-free states.

In field-free theory, first-order processes cannot exist due to momentum conservation, but in a strong magnetic field, transverse momentum is no longer conserved. The Feynman diagram for first-order magnetic electron-positron annihilation is shown in Figure 1. Here the electron and positron states (denoted by double lines) are solutions of the Dirac equation with an external magnetic field and are classified by the quantum numbers of longitudinal momentum $p_{z}$, Landau number $N$, and magnetic polarization $\tau$. The $S$-matrix element for this process reads

$$
\left[S_{f i}^{(1)}\right]_{\text {p.a. }}=i e \int_{-\infty}^{\infty} d^{4} x\left(\Psi_{N^{\prime}, p_{z}^{\prime}}^{-r^{\prime}}\right)^{*} M\left(\Psi_{N, p_{z}}^{+\tau}\right) e^{-i(\mathbf{k x}-\omega t)}
$$




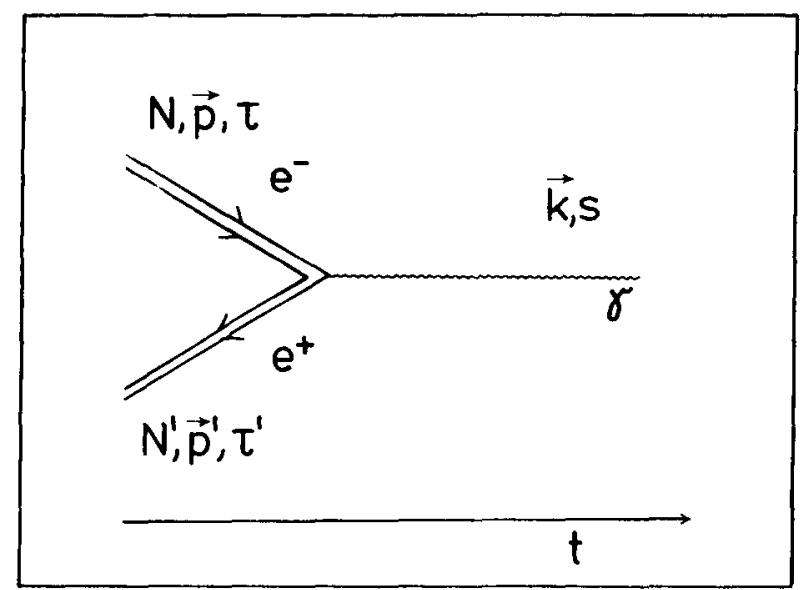

FIG. 1.-Feynman diagram for magnetic one-quantum annihilation

Here $M$ denotes the so-called polarization matrix, which depends on the polarization of the photon. The decay rate is obtained by squaring the $S$-matrix element and summing over all corresponding initial and final states. Here we are interested in the polarization-dependent rates. The cross section usually is normalized with respect to the cross section of the process $e^{+} e^{-} \rightarrow 2 \gamma$ without external field as a natural unit. Of course, it would be better to compare our results with the $2 \gamma$ cross section in an external magnetic field, which will be much smaller for higher Landau levels than that found for the Landau ground state $N=0$. Since nobody has done these calculations for higher Landau states up to now, a direct comparison with the corresponding $2 \gamma$-annihilation process in a strong magnetic field is not yet possible. In the Landau ground state, the $1 \gamma$-annihilation rate outweighs the $2 \gamma$-rate at magnetic field strengths of more than $0.24 B_{\text {cr }}$ as shown in Wunner (1979). We expect, however, $1 \gamma$-annihilation to become dominant also in higher Landau levels at magnetic field strengths of this magnitude.

The previous considerations allow us to determine the physi-

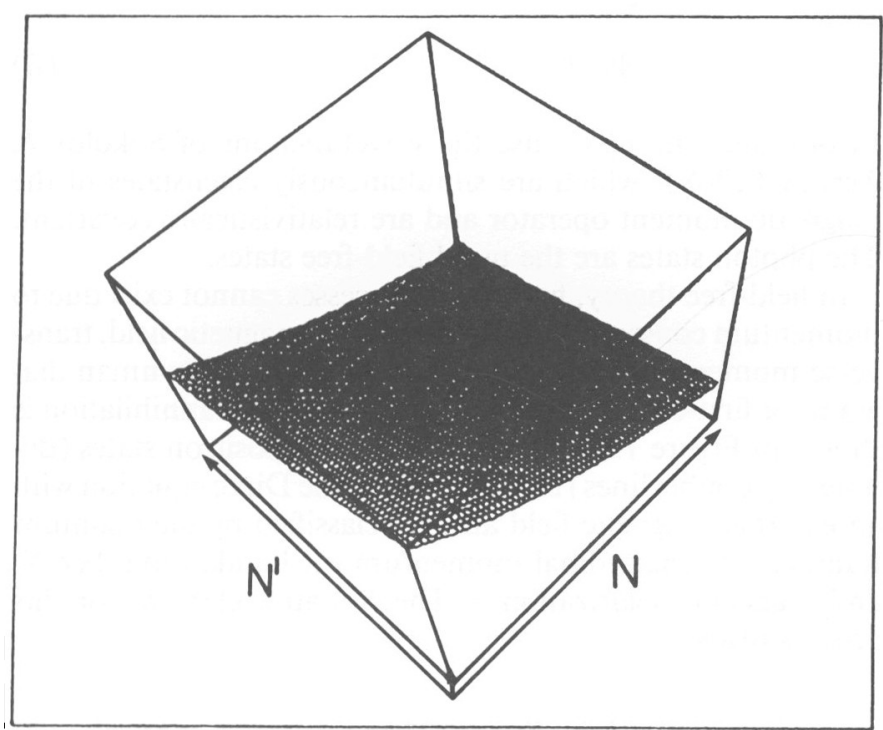

Fig. 2.-Cross section for antiparallel magnetic spin polarization for the first 100 Landau quantum numbers, $B / B_{\mathrm{cr}}=0.6, p / m=1.0$.

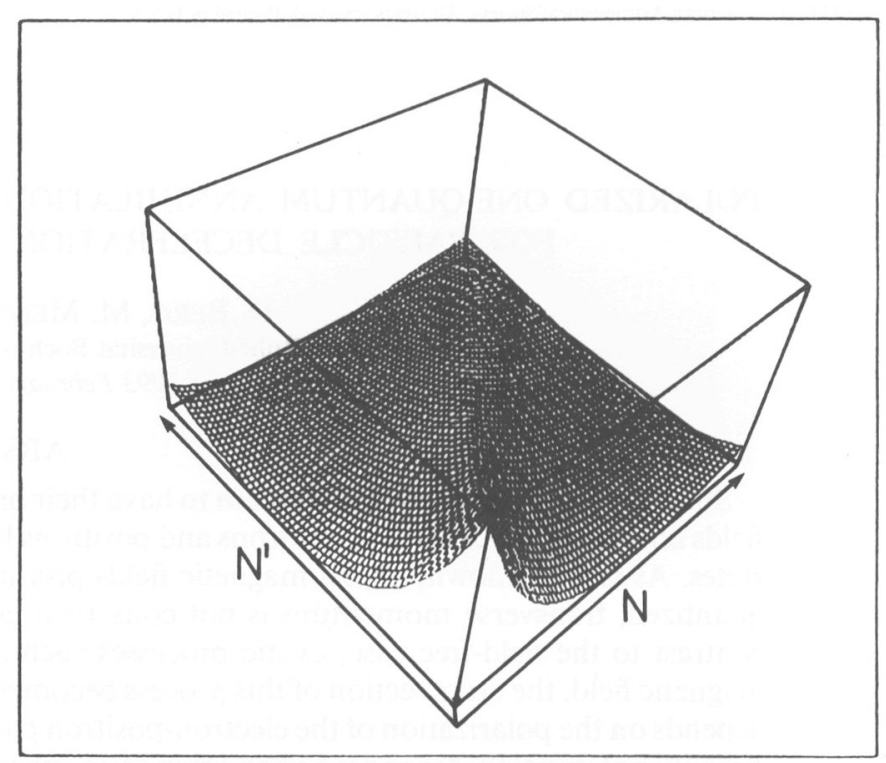

FIG. 3.-Cross section for parallel magnetic spin polarization with photon polarization parallel to magnetic field $B / B_{\mathrm{cr}}=0.6, p / m=1.0$.

cal observables from the $S$-matrix either exactly or approximately in the saddle point expansion (Klepikov 1954). The results coincide for higher Landau-quantum numbers.

In Figures 2 and 3 the polarized cross sections for some special polarizations are plotted versus the first 100 quantum
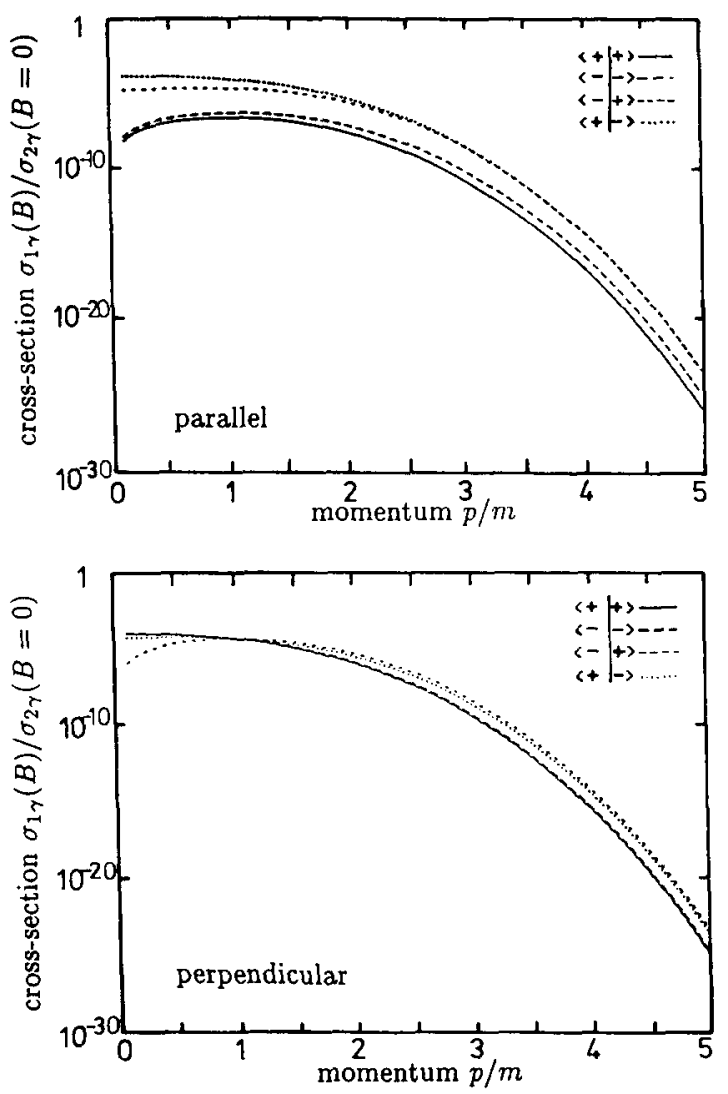

FIG. 4.-Momentum-dependent cross section for the different magnetic spin polarizations with photon polarization parallel (top) or perpendicular (bottom) to the magnetic field $B / B_{\mathrm{cr}}=0.4, N=90, N^{\prime}=100$. 


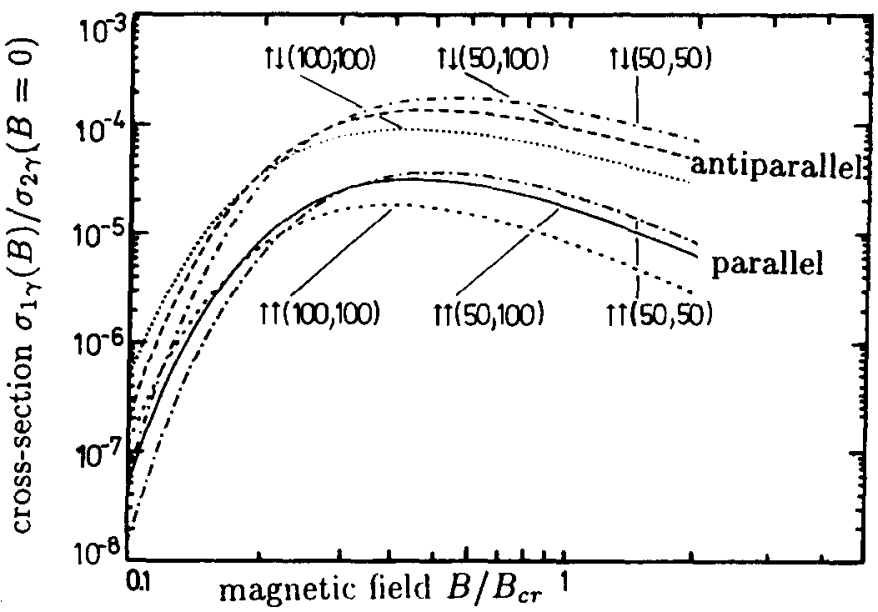

FIG. 5.-Polarized cross section vs. magnetic field for different pair quantum numbers, $p / m=1.0$.

numbers of electron and positron. An important feature is the symmetry between electrons and positrons. As one can clearly see, all cross sections are symmetric in $N$ and $N^{\prime}$. In Figure 2 the cross section for antiparallel magnetic spin polarization between electron and positron is shown. For other values of $B$ and $p$, the shape of the cross sections is similar to this one, but in some cases the cross sections show significant structures, mainly when the electron positron pair has a parallel magnetic spin polarization and the photon is emitted with polarization parallel to the magnetic field. An example is the cross section shown in Figure 3, which exhibits a type of interference for the case of identical Landau quantum numbers.

Figure 4 shows the momentum-dependent cross sections for each possible magnetic spin polarization of electron and positron. These are decomposed into two cases, namely whether the emitted photon is polarized parallel or perpendicular to the magnetic field. All cross sections decrease rapidly for higher momenta. In most cases cross sections with antiparallel spins of the electron-positron pair are larger than those with parallel spins. The difference between parallel and antiparallel polarization becomes more significant when the emitted photon is polarized parallel to the magnetic field.

In Figure 5 we show the polarization-dependent cross sections versus magnetic field for different pairs of Landau quantum numbers. In the region of known neutron star magnetic fields $\left(B / B_{\mathrm{cr}}=0.2 \cdots 0.6\right)$, the cross sections are maximum. As $B / B_{c r}$ tends to zero, all cross sections vanish; one-quantum annihilation does not exist in the free case. Again, antiparallel spin polarization yields higher cross sections.

In Figure 6 the polarization index

$$
P=\frac{|\sigma(\uparrow \uparrow)-\sigma(\uparrow \downarrow)|}{\sigma(\uparrow \uparrow)+\sigma(\uparrow \downarrow)}
$$

is plotted versus magnetic field for different pairs of quantum

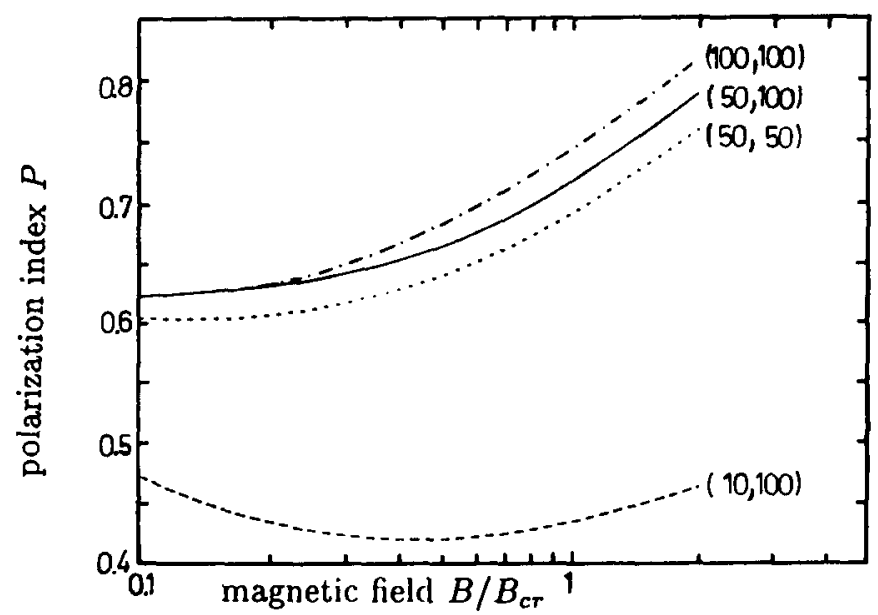

Fig. 6.-Polarization index vs. magnetic field for different pair quantum numbers, $p / m=1.0$.

numbers. Here, $\uparrow \uparrow$ denotes parallel electron positron spin polarization, $\uparrow \downarrow$ means antiparallel magnetic spin. $P$ assumes values in the interval $[0,1] . P=0$ means that there are no polarization effects, $\sigma(\uparrow \uparrow)=\sigma(\uparrow \downarrow)$. If $P=1$, one polarization is suppressed, and either $\sigma(\uparrow \uparrow)$ or $\sigma(\uparrow \downarrow)$ vanishes. Here, in most cases $P>0.5$, which means that all cross sections show strong polarization features. With growing magnetic field, the polarization index rises exponentially. The polarization effects increase with growing quantum numbers and are maximum when the quantum numbers for electron and positron are equal. On the other hand, if electron and positron Landau numbers differ much, one obtains a smaller polarization index.

In a magnetized electron-positron plasma, energy states are distributed over momenta and Landau-quantum numbers. One than has to integrate over a distribution function to obtain the temperature-dependent cross sections for an electron-positron plasma. In the case of a Fermi-distribution, for example, with $k T \simeq 1 \mathrm{MeV}$, the first 200 Landau states would be occupied.

Even if integrated in this sense over distributions of electronpositron pairs, the cross sections show strong dependence on polarization. It will be interesting to study polarization features for several distribution functions of pair momenta and quantum numbers. This work is in progress and will be published in a following paper, since the temperature-dependent cross sections are not model-independent like the cross sections mentioned here. Up to now, we have made no model assumptions. In a further paper we will discuss the pure quantum-electrodynamical processes in connection with some type of neutron star models. In contrast to the cross sections of the elementary process presented here, these results will be model dependent, and we therefore relegate the detailed discussion of temperature-dependent $1 \gamma$-annihilation rates and their application to the modeling of neutron star spectra to a subsequent paper.
Harding, A. K. 1991, Phys. Rep., 206, 327

Herold, H., Ruder, H., \& Wunner, G. 1981, Plasma Phys., 23, 775

Klepikov, N. P. 1954, Zh. Eksper. Teoret. Fiz., 26, 19

Mazets, E. P., et al. 1981, Ap\&SS, 1, 205

\section{REFERENCES}

Melrose, D. B., \& Parle, A. J. 1983, Aust. J. Phys., 36, 775

Sokolov, A. A., \& Ternov, I. M. 1968, Synchrotron Radiation (Berlin: Akademie)

Wunner, G. 1979, Phys. Rev. Lett., 42, 79 